\title{
The Effect of Low Level Laser with Repositioning Appliance in the Management of Temporomandibular Joint Disc Displacement with Reduction
}

\author{
Nermeen A. Rady ${ }^{1}$, Ahmed M. Abdelhamid ${ }^{2}$, Mahmoud K. Abd El-Razik \\ ${ }^{1}$ Assistant Lecturer of Alexandria University, Faculty of Dentistry, Alex, Egypt \\ ${ }^{2}$ Professor of Prosthodontics, Alexandria University Faculty of Dentistry, Alex, Egypt \\ ${ }^{3}$ Professor of Prosthodontics, Alexandria University Faculty of Dentistry, Alex, Egypt
}

\begin{abstract}
Statement of problem: Management of temporomandibular joint (TMJ) Disc Displacement with Reduction (DDwR) is controversial and always presented a therapeutic challenge. The literature suggested the use of a variety of physical therapies in conjunction with occlusal appliances. The majority of the studies were evaluating low Level Laser Therapy (LLLT) effect in treatment of patients with Temporomandibular Disorders (TMDs) of myogenic origin. Few studies had investigated its efficacy in TMDs of arthrogenic origin and degenerative disorders. Purpose: Evaluate of the effectiveness of LLLT as an adjunctive treatment modality for DDwR with Anterior Repositioning Appliance (ARA) in comparison to ARA alone. Materials and Methods: Thirty two female patients complaining of symptomatic clicking of TMJ were selected. They were diagnosed clinically using Research Diagnostic Criteria of Temporomandibular Disorders (RCD/TMD) as Axis I group II.a (DDwR) and confirmed by magnetic resonance imaging (MRI). They were randomly divided into 2 groups; the first group received maxillary ARA, while the second group received a combination of maxillary ARA and LLLT. Both groups were evaluated after three months for recapturing of articular disc by MRI and for subjective DDwR improvement in terms of Temporomandibular Index (TMI). Results: Clinical results revealed a significant reduction in the total TMI index in both groups $(P=0.001$ for both groups). Greater improvement from DDwR symptoms was noticed in group II (Who received LLLT and ARA), $87.5 \%$ of patients showed a complete relief of symptoms. However, in group I (who received ARA alone) 62.5\% of patients only showed a complete relief of their symptoms. Magnetic Resonance Imaging results displayed 62.5\% of group I patients and 87.5\% of group II patients showed recapturing of TMJ disc ( $p=0.001$ for both groups), concentric condylar position, and a mean decrease in anterior joint space values and a mean increase in posterior space values $(p=0.012,0.001$ for group I and II respectively). Conclusions: The combined treatment modalities used in this study resulted in recapturing of the articular disc and significant relief of the symptoms. In view of the noninvasive action of LLLT, it can be considered as an effective physical modality in the management of DDwR through its analgesic, anti inflammatory, and biostimulating effect.
\end{abstract}

Keywords: Temporomandibular Disorders, Internal Derangement, Disc Displacement with reduction, Low Level laser Therapy, Anterior Repositioning Appliances

\section{Introduction}

Temporomandibular disorders embrace a number of clinical conditions that involve the masticatory musculature, TMJ and associated structures. [1] The three most common TMDs are myofascial pain, internal derangement (ID) of the condyle-disc complex, and degenerative joint disease. [2] Internal derangements are the most common noninflammatory disorder of the TMJ and are defined as an abnormal relationship between the articular disc and the condyle. [3] Numerous factors have been proposed including traumatic events, bruxism, changes in the composition of synovial fluid, an improper activity of the lateral pterygoid muscle, and psychological stress. [1],[4],[5) Anterior DDwR is a commonly seen subgroup of ID, in which the intra-articular disc is positioned more anteriorly due to elongation of the inferior retrodiscal lamina and discal collateral ligament. The condyle rests on a more posterior portion of the disc resulting in abnormal translatory shift of the condyle over the disc during opening. However, the patient can manipulate the jaw as to reposition the condyle again onto the posterior border of the disc "Reducible disc". [6],[7] Various cardinal signs and symptoms are associated with DDwR as joint pain, clicking of joint, tenderness in masticatory muscles, and limitation in mandibular movements. [1]

During the last 35 years, the dental profession's attitude toward management of Disc Displacements (DDs) has changed greatly. [8] A wide consensus has been reached through the years on considering conservative and reversible approaches as first-line therapy of symptomatic DD including physical therapy, pharmacologic therapy, psychological counseling, cognitive behavioral therapy, treatment of parafunctional habits, occlusal appliances and acupuncture. Each of these treatment modalities can be used individually or may be combined. [9]

Occlusal appliance therapy is performed using various types of stabilization, anterior repositioning or bite plane appliances. [11] Moreover, numerous forms of physical therapy intervention, including ultrasound and transcutaneous electrical nerve stimulation, and Laser can be potentially effective for management of DD. These interventions are commonly employed to reduce pain and improve the mandibular range of motion [12]. 


\section{International Journal of Science and Research (IJSR) \\ ISSN (Online): 2319-7064}

Index Copernicus Value (2013): 6.14 | Impact Factor (2015): 6.391

A non traumatic introduction to dentistry can be represented by LLLT as an effective treatment modality of TMDs through its analgesic and anti- inflammatory effects. [13] However, the majority of researches [14] - [17] on the LLLT were evaluating its effect in treatment of patients with TMD of myogenic origin. Few studies [18],[19] had investigated the efficacy of LLLT in TMD of arthrogenic origin such as DDs. Therefore, the purpose of the present study was to evaluate the efficacy of using LLLT as an emerging trend along with ARA in comparison to ARA alone in patients suffering from DDwR.

\section{Material and Methods}

\subsection{Patient selection criteria and allocation}

Thirty two female patients were selected among consecutive patients who presented at the Removable Prosthodontics Department Clinic, Faculty of Dentistry, Alexandria University with TMJ pain and clicking.

Inclusion criteria were a chief complaint of TMJ pain during palpation, during function and/or during unassisted or assisted mandibular opening on at least one side, the presence of joint clicking during jaw opening and/or closing that was eliminated on protrusive opening, age range between 20 and 40 years, presence of full or nearly full complement of periodontally healthy natural teeth, Angle class I maxillo-mandibular relation.

Exclusion criteria were the presence of systemic diseases affecting the masticatory apparatus, bone, and joints (ex. rheumatoid arthritis, osteoarthritis, and epilepsy), history of recent oral, facial or cervical trauma, patients having nonreducible DD, myofascial pain, or degenerative joint disease detected by MRI, patients contraindicated to MRI examination such as implanted electronic devices, intracranial vascular clips, claustrophobia, metal particles in the eye or other vital structures, and pregnancy, and any therapeutic co-interventions during treatment.

The study was performed after gaining the approval of the Research Ethics Committee, faculty of Dentistry, Alexandria University. Patients were informed about the research procedures and follow-up examinations. Informed consent was filled out by each patient after a full explanation of the clinical trial.

\subsection{Therapeutic intervention}

Before treatment, all patients were clinically examined according to the RDC/TMD criteria by an experienced blinded prosthodontist. [20] We included patients with a RDC/TMD Axis I Group IIa who having DDwR then confirmed by MRI (Figure1). The patients were randomly divided into two equally groups. Those in group I $(n=16)$ underwent maxillary ARA therapy, those in group II $(n=16)$ were given LLLT as a physical modality in conjunction with maxillary ARA.

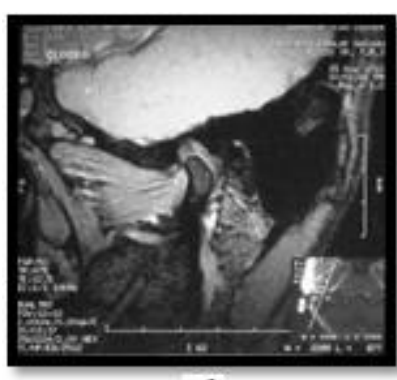

a)

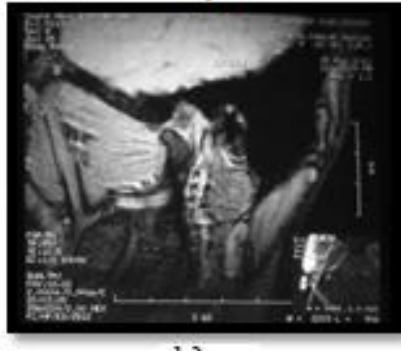

b)

Figure 1: Sagittal MRI showing right TMJ disc in a) closed and b) open mouth position revealed an anterior DDwR.

A maxillary full-arch hard clear acrylic ARA was fabricated according to Okeson's technique. [6] The degree of protrusion was the minimum necessary to eliminate joint clicking. The patients were instructed to use the appliance during night only for three months. (Figure 2)

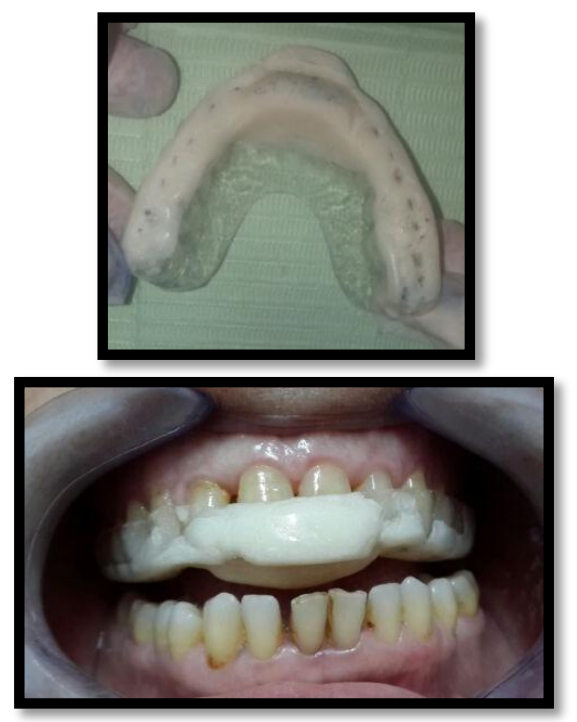

Figure 2: Maxillary Anterior Repositioning Appliance

The physical therapy protocol was conducted using Endolaser (Gallium-Aluminum- Arsenide. ENDOLASER 476, Enraf Nonius, Netherlands.). This low level diode Laser emits a continuous red Laser beam of $780 \mathrm{~nm}$ wavelength with the maximum power output $100 \mathrm{~mW}$ and energy density was adjusted to $1.4 \mathrm{~J} / \mathrm{Cm} 2$ for 1.30 minutes duration over the three trigger points at each TMJ; the posterior aspect of the joint, the area anterior to condyle approximately $1 \mathrm{~cm}$ in front of the tragus, and at the joint interface with the mouth opened. [13] The LLLT was performed three times per week for the entire treatment period. (Figure 3) 


\section{International Journal of Science and Research (IJSR) \\ ISSN (Online): 2319-7064}

Index Copernicus Value (2013): 6.14 | Impact Factor (2015): 6.391

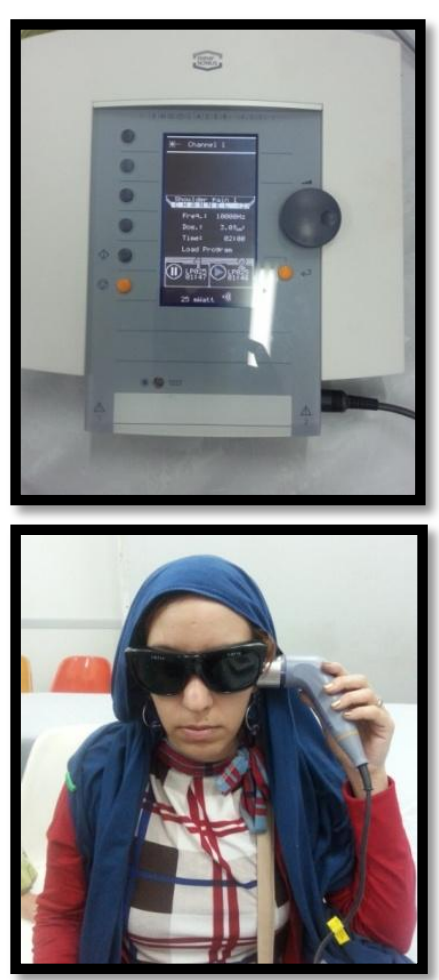

Figure 3: EndolaserTM 422, a low Level GaAlAs diode Laser

\subsection{Clinical evaluation}

All the patients were examined prior to, and at the end of treatment, by one blinded examiner. The baseline data were recorded at an initial examination before the start of treatment. The outcome data were collected immediately at the termination of all modalities (three months for both groups).

A subjective DDwR symptoms evaluation was made using the TMI as proposed by Pehling et al. [21] to record the severity of dysfunction (Figure) It has three sub-indices: 1function index (FI), 2- muscle index (MI), and 3- joint index (JI). The total index of the TMI is the mean of scores of the three sub-indices.

\subsection{Magnetic Resonance Imaging evaluation}

The "worest case" scenario of the pre-treatment MRI slices was selected and used for the reference image. Posttreatment sagittal MRIs of TMJs were made after three months for the two groups and compared with the reference image. Both disc position and joint space were measured.

Measurement of disc position was performed using the method of Kurita et al [22], of measuring the relative and absolute distances of reference points; a tangent was drawn between the lowest part of the articular eminence $(\mathrm{T})$ and the highest edge of the external auditory canal $(\mathrm{P})$, a line was drawn perpendicular to the tangent, touching the posterior edge of the disc, and their intersection was marked as point D. The distances from point $\mathrm{T}$ to $\mathrm{P}$ (TP) and to $\mathrm{D}$ (TD) were measured and recorded in millimeters. Then, the relative location of the disc to TP was calculated as TD/TP and expressed in one-hundredths of the distance between points $\mathrm{T}$ and $\mathrm{P}$.

Measurements of anterior and posterior joint spaces were performed using the method of Rammelsberg et al. [23]. The center of the condyle was determined by determining the center of a circle which was drawn to closely approximate the condyle outline using the software program. A reference line parallel to the Frankfort plane was drawn through the center of the condyle. Because the heads of the patients were placed parallel to the Frankfort plane, the upper and lower image margins of sagittal tomograms were parallel to this plane. The area of the superior, anterior, and posterior joint space within an angle of 90 degree, 45 degrees anterior and 45 degrees posterior respectively was calculated between the condyle and the temporal fossa by using the margins with the lowest signal intensity of fossa and condyle. The position of the condyle, depending on the anterior and posterior joint space, was calculated as a Joint Space Index (JSI), as follows: JSI= [(post-ant)/ (post/ant) $] \times 100$. A positive value indicated anterior condylar position within the glenoid fossa and negative value demonstrated posterior condylar displacement within the glenoid fossa. While the zero value showed the centric condylar position.

\section{Results}

\section{Statistical analysis}

Data were fed to the computer and analyzed using IBM SPSS software package version 20.0. Student t-test was used to compare two groups for normally distributed quantitative variables, while Paired t-test was assessed for comparison between two periods. Mann Whitney test was used to compare between two groups for abnormally distributed quantitative variables and Wilcoxon signed ranks test to compare between two periods. Significance of the obtained results was judged at the $5 \%$ level.

\subsection{Comparison between the two studied groups according to total joint Index}

A significant decrease in the total TMI index was observed in both studied groups at the evaluation time in comparison to the baseline data ( $\mathrm{p}=0.001$ for both groups) However, the difference between group I and II did not reach a significant level $(\mathrm{p}=0.168)$. (Table1).

\subsection{Comparison between the two studied groups according to disc position}

There was a significant improvement of the disc position in both studied groups at the evaluation time $(\mathrm{p}=0.001$ for both groups) but there was no significant difference observed when compared between the two studied groups $(\mathrm{p}=0.101)$. (Table1).

\subsection{Comparison between the two studied groups according to JSI}

A significant increase in the JSI at the evaluation time compared to the baseline data in both studied groups $(\mathrm{p}=$ $0.012,0.001$ for group I and group II respectively. However, 


\section{International Journal of Science and Research (IJSR) \\ ISSN (Online): 2319-7064}

Index Copernicus Value (2013): 6.14 | Impact Factor (2015): 6.391

there was no significant difference when compared the two studied groups $(\mathrm{p}=0.092)$. (Table1).

Table 1: Comparison between the two studied groups according to Total TMI, Disc position and JSI

\begin{tabular}{|c|c|c|c|}
\hline & $\begin{array}{c}\text { Group I } \\
(\mathbf{n = 1 6})\end{array}$ & $\begin{array}{c}\text { Group II } \\
(\mathbf{n = 1 6})\end{array}$ & $\mathbf{p}_{\mathbf{1}}$ \\
\hline \multicolumn{4}{|c|}{ Total TMI } \\
\hline Before & $0.68(0.54-1.0)$ & $0.70(0.54-0.97)$ & 0.910 \\
\hline After & $0.03(0.0-1.0)$ & $0.0(0.0-1.0)$ & 0.168 \\
\hline $\mathbf{p}_{\mathbf{2}}$ & $0.001^{*}$ & $0.001^{*}$ & \\
\hline \multicolumn{4}{|c|}{ Disc position } \\
\hline Before & $31.54 \pm 4.58$ & $32.27 \pm 5.04$ & 0.671 \\
\hline After & $42.65 \pm 11.35$ & $49.37 \pm 11.06$ & 0.101 \\
\hline $\mathbf{p}_{\mathbf{2}}$ & $0.001^{*}$ & $0.001^{*}$ & \\
\hline \multicolumn{4}{|c|}{ JSI } \\
\hline Before & -33.33 & -33.33 & 0.376 \\
\hline After & $(-42.86--14.29)$ & $(-42.86--14.29)$ & 0.092 \\
\hline $\mathbf{p}_{\mathbf{2}}$ & $0.0(-60.0-0.0)$ & $0.0(-42.86-0.0)$ & $0.001^{*}$ \\
\hline
\end{tabular}

\section{Discussion}

Arthralgia and joint clicking are the most common complaints in patients seeking treatment for DDwR. The joint pain originates from elongation of the discal and capsular ligaments and/or compression of the retrodiscal tissues. [20] Temporomandibular joint clicking seems to be related to ligament problems and condyle-disc assembly alterations during jaw movement. [24] In this study, only patients with joint pain and clicking were included. As recapturing the disc on the condyle was the goal of treatment, we use MRI for determining the disc position. The patients in the two studied groups showed a significant improvement of subjective pain intensity, muscle tenderness, and joint sounds suggested that LLLT could be used as an adjunctive treatment for amelioration of pain and dysfunction with nighttime use of ARA.

Anterior repositioning appliances are designed for treatment of patients with IDs of the TMJ. Protrusion of the mandible may decrease overload on the retrodiscal tissues, allowing adaptive changes to occur. Several studies have revealed the short-term efficacy of this modality for reducing TMD symptoms, [25]-[27] in agreement with our present results.

Low level laser therapy has been applied clinically for the management of pain of myofascial and neurologic origin. This modality has been employed in patients with TMD, in view of its analgesic and muscle-relaxing effect, giving positive results. [16],[17]

In a prospective randomized study, Ismail et al. demonstrated that, as well as occlusal appliance therapy, physical therapy in combination with splint therapy was able to improve the VAS score and mandibular mobility of patients with arthrogenic TMD, [21] in agreement with our present results. Multidisciplinary treatment of patients with DDwR has been reported to eliminate DDwR symptoms. [23]

Full-time use of ARAs may be associated with adverse effects such as posterior open bite, occlusal alterations, and myostatic contracture of the inferior lateral pterygoid muscle. $[1],[26]$ Conti et al. reported that long-term partial use of these appliances had no adverse effects. [9] In the present study, no such side effects were observed in the groups treated with ARAs after three months. Okeson suggested that part-time use of an ARA is the final definitive treatment for DDs and disc dislocations with reduction. [1] As an anterior repositioning appliance can change the position of the mandible temporarily to enhance adaptation of the retrodiscal tissues with no side effects, this appliance was selected for the present study.

The results of the present study indicate that ARAs provides an improvement of TMD and that therefore it is a useful approach for reduction of pain levels and joint sounds. As significant improvement was also observed in the group II including LLLT, this modality can be used as a conservative low-cost intervention.

\section{Conclusion}

Within the limitations of this study regarding the short study periods; it is possible to conclude that a multidisciplinary management approach should be considered for the optimal treatment of disc displacement with reduction including both oral appliances and physical modalities. After the application of a disc-repositioning appliance and Low Level Laser Therapy, reduction in the position of an anteriorly located disc was observed. However, in order to determine whether the corrected disc-condyle relationship is stable, treated patients must be followed longitudinally.

\section{References}

[1] Khan M, Vijayalakshmi KR, Gupta N. Low intensity laser therapy in disc derangement disorders of temporomandibular joint: A review article. Int $\mathbf{J}$ Odontostomat 2013;7(2):235-239.

[2] Detamore MS, Athanasiou KA, Mao J. A call to action for bioengineers and dental professionals: directives for the future of TMJ bioengineering. Ann Biomed Eng 2007; 35(8):1301-1311.

[3] Laskin DM. Temporomandibular disorders an evidencebased approach to diagnosis and treatment. Chicago: Quintessence Publishing Co Inc; 2006.

[4] Nitzan DW. The process of lubrication impairment and its involvement in temporomandibular joint disc displacement: a theoretical concept. J Oral Maxillofac Surg 2001; 59:36-45.

[5] Yun PY, Kim YK. The role of facial trauma as a possible etiologic factor in temporomandibular joint disorder. J Oral Maxillofac Surg 2005; 63:1576-1583.

[6] Okeson J.P: Management of temporomandibular disorder and occlusion, 6th ed. Elsevier Mosby Co, St. Louis, 2008. pp 176-189,405,408,413.

[7] Hatice Go“" k, Hakan Tu“ R. Changes in Position of the Temporomandibular Joint Disc and Condyle After Disc Repositioning Appliance Therapy: A Functional Examination and Magnetic Resonance Imaging Study. Angle Orthod 2000; 70:400-408.

[8] Stohler CS, Zarb GA. On the management of temporomandibular disorders: a plea for a low-tech, high-prudence therapeutic approach. J Orofac Pain $1999 ; 13: 255-261$.

[9] [9] Eberhard D, Bantleon HP, Steger W. The efficacy of 


\section{International Journal of Science and Research (IJSR) \\ ISSN (Online): 2319-7064 \\ Index Copernicus Value (2013): 6.14 | Impact Factor (2015): 6.391}

anterior repositioning splint therapy studied by magnetic resonance imaging. Eur J Orthod 2002; 24:343-52.

[10] Petrucci A, Sgolastra F, Gatto R, Mattei A, Monaco A. Effectiveness of low-level laser therapy in temporomandibular disorders: a systematic review and meta-analysis. J Orofac Pain 2011; 25(4):298-307.

[11] Hanefi K, Emre M, Tonguc S, Demirhan D, Fatma U, Cihan A, Necat T. Comparison of Effectiveness of Stabilization Splint, Anterior Repositioning Splint and Behavioral Therapy in Treatment of Disc Displacement with Reduction. Turk J Phys Med Rehab 2011; 57:2530.

[12] McNeely ML, Armijo Olivo S, Magee DJ. A systematic review of the effectiveness of physical therapy interventions for temporomandibular disorders. Phys Ther 2006; 86, 710-725.

[13] Venancio RA, Camparis CM, Lizarelli RF. Low intensity laser therapy in the treatment of temporomandibular disorders: a double-blind study. J ral Rehabil 2005;32(11):800- 7.

[14] Afifi M. The use of low intensity laser in conjunction with occlusal splint therapy as a symptomatic treatement of temporomandibular joint disorders. M.Sc. Thesis. Faculty of Dentistry, Alexandria University.2009.

[15] Hakgüder A, Birtane M, Gürcan S, Kokino S, Turan FN. Efficacy of low level laser therapy in myofascial pain syndrome: an algometric and thermographic evaluation. Lasers Surg Med 2003; 33(5):339-43.

[16] Arash A, Mahnaz S, Shirin L, Fereydoon J, Nader M. Effects of Low-level Laser in the Treatment of Myofascial Pain Dysfunction Syndrome JODDD 2007; $1(2)$.

[17] Shinozaki EB, Santos MB, Okazaki LK, Marchini L, Junior AB. Clinical assessment of the efficacy of low level laser therapy on muscle pain in women with temporomandibular dysfunction, by surface electromyography. Braz J Oral Sci 2010; 9(4):434-8.

[18] Bertolucci LE, Grey T. Clinical analysis of mid-laser versus placebo treatment of arthralgic TMJ degenerative joints. J Craniomand Pract 1995; 13:26 - 9.

[19] Bertolucci LE, Grey T. Clinical comparative study of microcurrent electrical stimulation to mid-laser and placebo treatment in degenerative joint disease of the temporomandibular joint. J Craniomand Pract 1995; 13:116-20.

[20] Dworkin SF, LeResche L. Research diagnostic criteria for temporomandibular disorders: review, criteria, examinations and specifications, critique. J Craniomandib Disord 1992;6, 301-355.

[21]Pehling J, Schiffman E, Look J, Shaefer J, Lenton P, Fricton J. Interexaminer reliability and clinical validity of the temporomandibular index: a new outcome measure for temporomandibular disorders. J Orofac Pain. 2002;16:296-304

[22] Kurita H, Kurashina K, Ohtsuka A, Kotani A. Change of position of the temporomandibular joint disc with insertion of disc-repositioning appliance. Oral Surg Oral Med Oral Pathol Oral Radiol Endod 1998;85:142-5.

[23] Rammelsberg P, Jager L, Duc J. Magnetic resonance imaging-based joint space measurements in temporomandibular joints with disc displacements and in controls. Oral Surg Oral Med Oral Pathol Oral Radiol Endod 2000;90:240-8.
[24] Conti PC, Miranda JE, Conti AC, Pegoararo LF, Araújo Cdos R. Partial time use of anterior repositioning splints in the management of TMJ pain and dysfunction: a oneyear controlled study. J Appl Oral Sci 2005;13, 345-350.

[25] Mazzeto MO, Hotta TH, Mazzetto RG. Analysis of TMJ vibration sounds before and after use of two types of occlusal splints. Braz Dent J 2009;20, 325-330.

[26] Williamson EH. Temporomandibular dysfunction and repositioning splint therapy. Prog Orthod 2005;6, 206213.

[27] Azam SM and Amirtaher M. Comparison of three treatment options for painful temporomandibular joint clicking. Journal of Oral Science 2011;53(3), 349-354

\section{Author Profile}

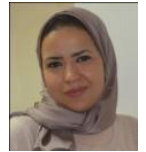

Nermeen Abd Elsalam Rady, received the B.D.S. in Dental and Oral Surgery from Alexandria University, faculty of dentistry 2004. Since 2006 till now, she is one of the staff in Removable prosthodontic Department, faculty of dentistry, Alexandria University, Egypt. During 2008-2011, she educated for M.S degree in prosthodontics in prosthetic department, faculty of dentistry. Alexandria University. During 2011-2016, she educated for PhD degree in prosthodontics in prosthetic department, faculty of dentistry. Alexandria University. 